\title{
Open Knowledge Representation Techniques and Its Application in Precision Forming Design
}

\author{
Ping Wang ${ }^{1, a}$, Xiao Gui Tang $^{2}$ and Bin Wang ${ }^{1}$ \\ ${ }^{1}$ School of Mechanical Engineering, Yancheng Institute of Technology, 224051 Yancheng Jiangsu, China \\ ${ }^{2}$ School of Mechanical Engineering, Jianghsu University, 212013 Zhenjiang Jiangsu, China
}

\begin{abstract}
Precision forming knowledge of precision forging parts can be acquired from various sources and generally requires an effective representation for its efficient reuse. According to the specific characteristics of the precision forming of car forging parts, the precision forming engineering knowledge has been classified into several kinds with the method of the open knowledge representation techniques for the different kinds of knowledge proposed. The open representation techniques of precision forming knowledge and its application are described in detail, and combined with the development of the precision forming design system of the automotive starter guiding cylinder based on knowledge. The results show that the precision forming knowledge is represented in an effective way will solve the problem of fusion of precision forming knowledge and precision forming manufacturing, and improve greatly the utilization efficiency of the precision forming knowledge.
\end{abstract}

\section{Introduction}

Knowledge-based Engineering (KBE) has been used widely, which integrates artificial intelligence with CAX system and connects engineering design without interruption [1]. KBE application has become pivotal for enhancing manufacturing enterprises competitiveness. Product design is a highly involved, often ill-defined, complex and iterative process [2]. An effective Knowledge driven design tool requires efficient knowledge representation schemes. Therefore, many scholars have carried out the research of knowledge representation. Lee and Jeong[3] developed a distributed and flexible knowledge representation model, and tested a prototype. Edy Portmann, Patrick Kaltenrieder and Witold Pedrycz[4] put forward knowledge representation through graphs and provided an overview of different types of graphs stressing their relationships and their essential features. Haining Yao and Letha Etzkorn[5] introduced a semantic converter which converts knowledge representation in Conceptual Graphs into representation in Resource Description Framework. W.M. Wang, X. Peng[6] presented a dynamic representation of fuzzy knowledge (DRFK) method. Chen, W.L[7] put forward a knowledge representation approach based on Parameter Flow Chart. Xi P[8] studied the open representation of design knowledge in the form of formaluas, 2D mapping graphs, procedures and rules.

The problem with these researchers is that few papers have addressed the open knowledge representation in intelligent precision forming design. In this paper, we provided an open knowledge representation and its application in precision forming design system. This paper is organized as follows: Section 2 describes the precision forming design process based on knowledge. Section 3 puts forward the open representation of precision forming knowledge. Section 4 presents an application implementation of the knowledge driven precision forming design combined with the automotive starter guiding cylinder. The conclusion and future work are discussed in Section 5.

\section{The precision forming design process based on knowledge}

Based on the characteristics of precision forming design, the precision forming design process based on knowledge is shown in Fig. 1.

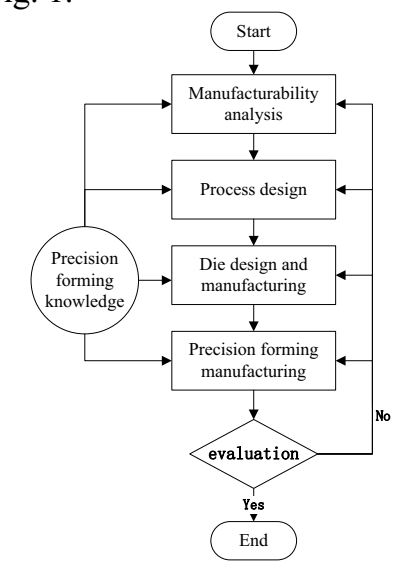

Figure 1. Precision forming design process based on knowledge.

\footnotetext{
$\overline{{ }^{a} \text { Corresponding author: wp_ycit@ } 163 . c o m}$
} 
Precision forming design, especially the precision forming design of complex product is a highly involved, often ill-defined, complex process in which design engineers perform a large number of activities related to creating and evaluating solutions. Most of these activities depend upon the design knowledge obtained in previous projects. In order to improve the efficiency and quality of the precision forming design of car forging parts, precision forming knowledge should be properly fused with forming design process.

\section{The open representation of precision forming knowledge}

According to characteristics of the precision forming of car cold/warm forging parts, the precision forming knowledge is usually classified into four categories, namely, process design knowledge, die design knowledge, numerical simulation knowledge and forming processing knowledge. As shown in Fig.2.

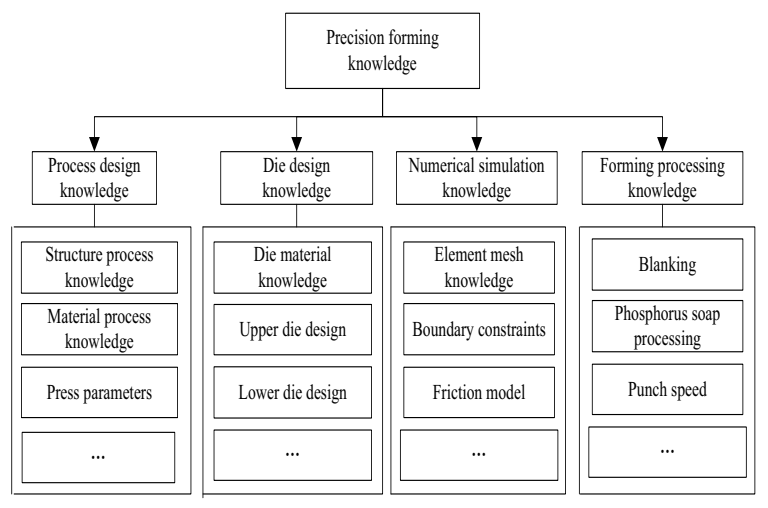

Figure 2. The classification of precision forming knowledge.

\subsection{The open representation of formulas knowledge}

There are many formula requires an effective representation for its efficient reuse in the process of precision forming design. The BNF (Backus Naur Form) representation is usually used to represent the knowledge. The paradigm is as following:

$<$ formula knowledge $>::=<$ variable $><$ expressions $>$

$$
\begin{aligned}
&<\text { variable }>::=<\text { variable name }> \\
&<\text { variable type }> \\
&<\text { variable value }> \\
&<\text { expressions }>::=<\text { item }>\mid
\end{aligned}
$$$$
(<\text { item }><\text { the operator }>)
$$

\subsection{The open representation of graphs knowledge}

Engineering graphs are common forms of precision forming knowledge. The BNF representation of graphs knowledge is described as following:

$<$ graph knowledge $>::=<$ query statement $><$ graph data $>$

$<$ query statement $>::=<$ graph $\mathrm{X}>\mid<$ graph $\mathrm{Y}>$

$<$ graph data $>::=<$ graph name $>$

$<$ original data $>$

$<\mathrm{X}$ data $><$ Y data $>$
3.3 The open representation of table knowledge Engineering table knowledge is usually stored in relational database, and the designers can easily query some parameter values with the help of query statements. The BNF representation of table knowledge can be described as following:

$<$ table knowledge $>::=<$ query statement $><$ table data $>$

$<$ query statement $>::=<$ query parameter $>$

$<$ table name $>$

$<$ parameter field $>$

$<$ conditions $>$

$<$ table data $>::=<$ table name $>$ fields $><$ records $>$

\subsection{The open representation of rule knowledge}

In the process of precision forming design, process rules are usually used to represent the empirical domain knowledge. The BNF representation of process rules is as follows:

$<$ rules $>::=$ IF $\{<$ conditions $>\}$ THEN $\{<$ conclusions $>\}$

$<$ conditions $>::=<$ variable $><$ predicate $>$ $<$ attribute value $>\mid<$ facts $>$

$<$ conclusions $>::=\{<$ statements $>\mid<$ operations $>\}$

$<$ statements $>::=\{<$ statement items $>\}$

$<$ operations $>::=\{<$ operation functions $>\}$

\subsection{The open representation of process knowledge based on fuzzy logic}

The fuzzy sets and membership function in fuzzy mathematics can be used to represent the uncertainty process knowledge in the precision forming design development.

If $U$ domain is numerical domain, and the process knowledge can be described by numerical values, the membership function of $U$ domain fuzzy subset can be defined as a linear function with parameters, namely:

$$
\mu_{A}(x, a, b, c, d)= \begin{cases}0 & x \leq a \\ \frac{x-a}{b-a} & a<x \leq b \\ 1 & b<x \leq c \\ \frac{d-x}{d-c} & c<x \leq d \\ 0 & d<x\end{cases}
$$

Where, a, b, c and d denote respectively design parameters, and a value is less than $b$ value, $b$ value is less than or equals to $\mathrm{c}$ value, $\mathrm{c}$ value is less than $\mathrm{d}$ value.

\subsection{The open representation of case knowledge}

In the process of precision forming design, there are much forming design support knowledge, such as part name, part material, part shape and similar parts forming design etc., the open representation of case knowledge is as follows:

CASE INSTANCE: Case_No

Name_of_part:

Material of Part:

MaterialType_of_part:

Shapetype_of_part: 


\section{SOLUTION}

Process file_Name:

Part_file_Name:

Part_file_path:

END

\subsection{The open representation of object-oriented precision forming knowledge}

The method of object-oriented knowledge representation provides the approach of integrating forming process knowledge into forming design. These methods include abstraction, encapsulation, inheritance and polymorphism. The open representation of object-oriented precision forming process knowledge is as follows:

Object:: $=$ (object name, data structure, the method sets, message interface)

\subsection{The open representation of precision forming knowledge based on artificial neural network}

For the open knowledge representation of multiple inputs and multiple outputs precision forming process knowledge, the BP neural network is usually adopted, as shown in Fig. 3.

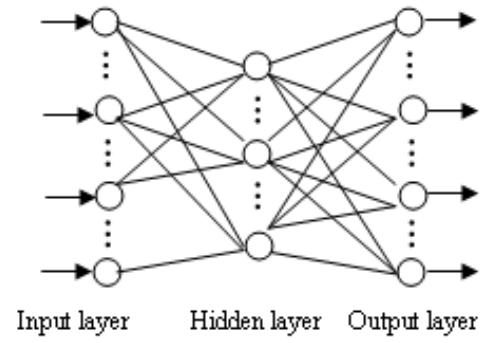

Figure 3. The BP neural network.

\section{Application case}

Automotive starter guiding cylinder (as shown in Fig.4) is the critical part of automotive starter transmission system, it works in a complex condition, and it has to meet the requirements for the excellent mechanical properties. At present, most automotive starter guiding cylinders are manufactured by precision forming, in which is characterized by low cost, high productivity, high precision and excellent mechanical properties. As there is so much design knowledge involved in the forming process, therefore, it can greatly improve the efficiency of the precision forming technology and die design to incorporate design knowledge and forming process, and realize the reuse and sharing of knowledge.

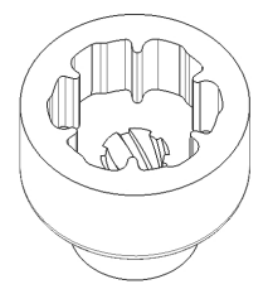

Figure 4. Automotive starter guiding cylinder.
This study proposes an open knowledge representation of the automotive starter guiding cylinder precision forming based on NX 7.5 software platforms with the help of NX/OPEN API tools, and realize the integration of precision forming design knowledge and precision forming design process. NXOPEN API is open programming interfaces of NX software. NX/OPEN API consists of C++ API, C\# API, VB.NET API and Java API. Fig. 5 shows the interface of the automotive starter guiding cylinder precision forming design system based on $\mathrm{KBE}$.

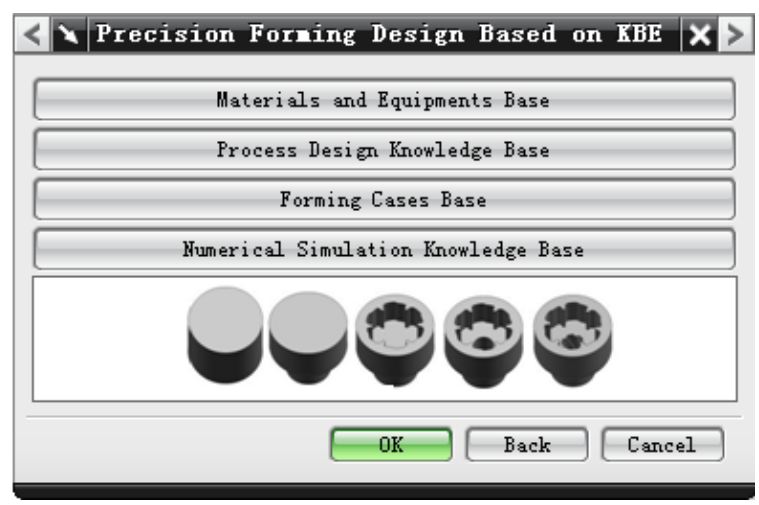

Figure 5.The interface of precision forming design based on KBE

\subsection{The open representation of precision forming design formula}

The complicated shape coefficient of extrusion $F_{k}$ is often checked in the precision forming design, and the formula of the complicated shape coefficient of extrusion is as follows:

$$
F_{K}=\frac{V_{0}}{V_{1}}
$$

Where, $\mathrm{V} 0$ is the preformed blank volume, $\mathrm{V} 1$ is the volume of final forming part.

The knowledge representation of formula (2) is as follows:

$$
\mathrm{FK}=(\mathrm{V} 0) /(\mathrm{V} 1)
$$

\subsection{The open representation of graphs in the precision forming design}

Engineering graphs are common forms of precision forming design knowledge. Fig. 6 is the Z-versus-stroke graph for the automotive starter guiding cylinder precision forming, where $\mathrm{Z}$ is the forming load, stroke is the extrusion displacement.

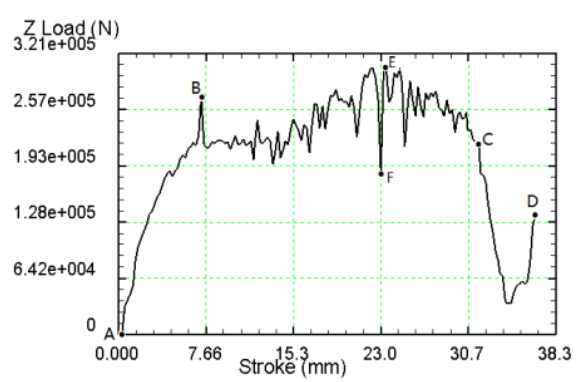

Figure 6. The Z-versus-stroke curve. 
The BNF representation of graphs knowledge is described as following:

$<$ graph knowledge $>::=<$ query statement $><$ graph data $>$

$<$ query statement $>::=<$ graph $\mathrm{X}>\mid<$ graph $\mathrm{Y}>$

$<$ graph data $>::=<Z$ Z_load_curve $>$

$<$ original data $><0.0><0.0>$

\subsection{The open representation of table knowledge in the precision forming design}

Table 1 is the parameters of the final forged part and the billet for the automotive starter guiding cylinder precision forming.

Table 1. The parameters of the final formed part and the billet.

\begin{tabular}{|c|c|c|}
\hline Parameter & Unit & Value \\
\hline $\begin{array}{c}\text { The diameter of billet guiding } \\
\text { hole }\end{array}$ & $\mathrm{mm}$ & 16 \\
\hline $\begin{array}{c}\text { The height of billet guiding } \\
\text { hole }\end{array}$ & $\mathrm{mm}$ & $34 \pm 0.5$ \\
\hline $\begin{array}{c}\text { Teeth number of helical } \\
\text { involute spline }\end{array}$ & $\mathrm{mm}$ & 120 \\
\hline $\begin{array}{c}\text { The lead of helical involute } \\
\text { spline }\end{array}$ & $\mathrm{degree}$ & 25.6611 \\
\hline Helical angle & $\mathrm{mm}$ & 1.37846 \\
\hline Normal module & $\mathrm{mm}$ & 16.609 \\
\hline Direction of turning & $\mathrm{mm}$ & 1.52929 \\
\hline Pitch diameter & $\mathrm{mm}$ & $20.635 \pm$ \\
\hline Circumferential module & 0.075 \\
\hline root diameter & $\mathrm{mm}$ & $16.949 \pm 0.15$ \\
\hline outside diameter & $\mathrm{mm}$ & 18.351 \\
\hline standard pitch diameter & $\mathrm{degree}$ & 30 \\
\hline Pressure angle &
\end{tabular}

The open representation of table 1 knowledge is as follows:

The_forming_parameters=Table ("The parameters of the final formed part and the billet", the forming parameters, the value of the parameter)

\subsection{The open representation of case knowledge in the precision forming design}

The case knowledge of automotive starter guiding cylinder mainly includes parts name, parts material, the extrusion speed of the punch, forming temperature, friction coefficient and the stress and strain distribution in the forming process. The open representation of case knowledge is as follows:

CASE INSTANCE: Case No. ASGC

Name_of_part: $\mathrm{ASGC}$

Material_of_Part: 20CrMo

MaterialType_of part: Metal

Shapetype_of_part: Axially Symmetrical Parts

Punch_speed: 12

Forming_temperature:20

\author{
SOLUTION ASGC \\ Process file_Name:ASGC process file \\ Part_file_Name:ASGC.stl \\ Part_file_path:D: $\backslash$ ASGC \\ END \\ ...
}

Fig. 7 shows the stress distribution of forming process in the forming case base.

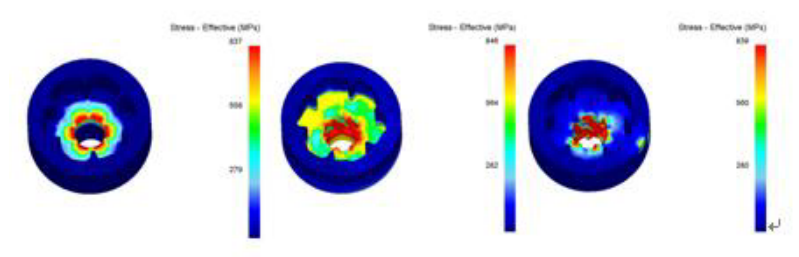

Figure 7. The stress distribution of forming process.

\section{Conclusions}

In this study, an open representation of precision forming design knowledge is proposed, and it can help capture design knowledge from various sources in the precision forming design process, and can effectively realize the fusion of precision forming design knowledge and precision forming process. The methods proposed are shown to be useful for precision forming knowledge reuse. In our future work, we will study how to enable designers to formulate complex knowledge queries so that relevant precision forming design knowledge can be recommended automatically.

\section{Acknowledgment}

The project was supported by joint innovation project of Jiangsu Province under Grant BY2015057-28.

\section{References}

1. J. Hu, Y.H. Peng, D.Y. Li, J.L.Yin, Robust optimization based on knowledge discovery from metal forming simulation, Journal of Materials Processing Technology, 187-188, 698-701(2007)

2. Chandrasegaran, Senthil K., et al. , The Evolution, Challenges, and Future of Knowledge Representation in Product Design Systems, Computer-Aided Design, 45,204-228(2013)

3. Lee, J. and Y. Jeong, User-centric Knowledge Representations Based on Ontology for AEC Design Collaboration",Computer-Aided Design 44,735748(2012)

4. Edy Portmann, Patrick Kaltenrieder and Witold Pedrycz, "Knowledge representation through graphs", Procedia Computer Science 62 ,245-248(2015)

5. Haining Yao, Letha Etzkorn, "Automated conversion between different knowledge representation formats", Knowledge-Based Systems, 19,404-412,(2015)

6. W.M Wang,W.M. Wang, X. Peng, Dynamic representation of fuzzy knowledge based on fuzzy 
petri net and genetic-particle swarm optimization, 41,1369-1376(2014)

7. Chen, W.L, A New Process Knowledge Representation Approach Using Parameter Flow Chart, Computers in industry, 62,9-22(2011)

8. Xi P et al., Intelligent Product Design Based on Open Knowledge Representation. Acta Aeronautica et Astronautics Sinica, 33,1746-1754(2012) 